\section{Bancbanus - ungarisch, österreichisch, kroatisch Zur Inszenierung eines umstrittenen historischen Stoffes im 19. Jahrhundert}

1.

Zu den historischen Stoffen, die von Autoren aus unterschiedlichen Völkern des Habsburgischen Reiches im 19. Jahrhundert literarisch transponiert und dabei mit deutlichen Hinweisen auf den gleichzeitig verlaufenden Entstehungsprozess moderner Nationen und zugehöriger (über)nationaler Narrative versehen wurden, gehört auch die Geschichte des ungarischen Aristokraten Bánk (in deutscher Überlieferung Bancbanus, in kroatischer Benko/Banko Bot). ${ }^{1}$ Den hohen Würdenträger, der verschiedene bedeutende Ämter zur Regierungszeit des Arpadenkönigs Andreas II. am Anfang des 13. Jahrhunderts bekleidet haben soll, so auch das Amt des kroatischen Banus (Statthalter), und der vom Monarchen wegen seiner außerordentlichen Treue mit dem Ehrentitel `fidelis` bedacht wurde, wählten drei namhafte Theaterautoren des 19. Jahrhunderts als Helden ihrer Bühnenwerke:

1 Der vorliegende Aufsatz geht auf einen Vortrag im Rahmen des Symposiums »Germanistik als Sprach-, Kultur- und Geisteswissenschaft. Der neue deutsche (Kolonial)roman in kulturwissenschaftlicher, interkultureller und postkolonialer Perspektive« an der Universität Lomé/Togo im April 2014 zurück. Eine kürzere Fassung ist erschienen in: Csire u.a. (Hgg.): Ein Land mit Eigenschaften, S. 225-237.
Im Beitrag werden drei Dramen-Versionen des Bancbanus-Stoffes im 19. Jh. dargestellt: der mittelalterliche ungarische Aristokrat Bánk (dt. Bancbanus, kroat. Benko Bot) erscheint als Held eines historischen Dramas bei dem Ungarn József Katona, bei dem Deutschösterreicher Franz Grillparzer und bei dem Kroaten Franjo Marković. Ihre unterschiedlichen ästhetisch-politischen Positionen lassen in der Darstellung des Stoffes dennoch ein übereinstimmendes Anliegen erkennen: vor dem Hintergrund der snation building -Prozesse in der Donaumonarchie und der damit zusammenhängenden Stärkung zentripetaler Tendenzen die Reflexion über den gemeinsamen (über)nationalen staatlichen Rahmen anzuregen. 
der Ungar József Katona (1791-1830) im Drama Bánk bán (E 1815/19, D 1820, UA 1833), der Deutschösterreicher Franz Grillparzer (1791-1872) im Trauerspiel Ein treuer Diener seines Herrn (E 1826, UA 1828, D 1830) und der Kroate Franjo Marković (1845-1914) im Trauerspiel Benko Bot (E, D 1872, UA 1899). So unterschiedlich die ästhetischen und politischen Positionen der drei Autoren auch sein mögen, so lassen ihre Darstellungen des Bancbanus-Stoffes, von denen im Folgenden die Rede sein wird, ein in vielerlei Hinsicht übereinstimmendes Anliegen erkennen: Vor dem Hintergrund der Nationsbildungs-Prozesse in der Donaumonarchie und der damit zusammenhängenden Stärkung zentripetaler Tendenzen haben sie nicht nur zum Ziel, ihrem Publikum ästhetischen Genuss zu bieten, sondern vor allem zur Reflexion über den gemeinsamen (über)nationalen staatlichen Rahmen anzuregen.

Der historische Stoff, auf dem die Dramatiker ihre Werke gründen, ist spärlich überliefert und bezieht sich auf die frühe Regierungszeit des Königs Andreas II. (1205-1235), als der ungarische Monarch durch Eroberungskriege sein Reich im Nordosten zu erweitern suchte. ${ }^{2}$ Ungarische Aristokraten, über die Bevorzugung fremdländischen Adels durch Andreas' Frau Gertrud aus dem bayerischen Geschlecht Andechs-Meranien empört, schlossen sich bei einer längeren Abwesenheit des Königs 1213 zu einer Verschwörung zusammen und töteten nicht nur die verhasste Königin, sondern auch eine Reihe ihrer ausländischen, v.a. deutschstämmigen Günstlinge; Gertruds Bruder Berthold, der wohl bekannteste unter den privilegierten fremdländischen Aristokraten, konnte sich jedoch retten.

Am Aufstand gegen die Königin soll sich neben dem Grafen Peter und dem Banus Simon, die nach der Rückkehr des Königs als Anführer der Verschwörung hingerichtet wurden, auch der Banus Bánk beteiligt haben; davon berichtet allerdings erst eine spätere Überlieferung. Dies deutet darauf hin, dass Bánk, dessen Name als Palatin und kroatischer Banus sowohl vor 1213 als auch einige Jahre danach in historischen Quellen erscheint, erst später der Mitschuld an der Ermordung der Königin bezichtigt wurde, da Andreas II. wohl kaum den Mörder seiner Gattin mit dem Ehrentitel >fidelis` und mit hohen staatlichen Ämtern ausgezeichnet hätte. Aus späteren Zeiten stammt auch die Behauptung, der eigentliche Grund für Gertruds Ermordung habe in der von ihr veranlassten oder zumindest geduldeten Schändung an Bánks Gattin gelegen.

Aus der Vermengung dieser unterschiedlichen, sich zum Teil auch widersprechenden Überlieferungen gingen die beiden zentralen Handlungs-

2 Zum historischen Hintergrund und zur Person Bánks vgl.: Görlich: Grillparzer und Katona, S. 128f.; Klaić: Povijest Hrvata, Bd. I, S. 223f.; Hrvatski biografski leksikon, Bd. I, S. 655. 
stränge der Bühnenwerke von Katona, Grillparzer und Marković hervor - der politische und der erotische. Der so entstandene Stoff verweist auch auf die grundsätzlich übereinstimmende Figurenkonstellation, in der sich allerdings von einem zum anderen Autor auch einige Abweichungen erkennen lassen: dem beeinflussbaren, letztlich aber doch weisen und gerechten König steht eine intrigante, herrschsüchtige und skrupellose Königin zur Seite; der alte Bánk wiederum wird grundsätzlich als ein verlässlicher und anhänglicher, manchmal allerdings impulsiv reagierender $>$ Diener seines Herrn bezeichnet oder beschrieben; Bánks viel jüngere Gattin schwankt wiederum zwischen der Treue zu ihrem Mann, den sie eher als eine väterliche Gestalt sieht, und der erotischen Faszination, die von Gertruds Bruder ausgeht, dessen Zudringlichkeiten - begleitet vom böswilligen Intrigenspiel der Königin - sie schließlich in den Tod treiben.

Die Verschränkung des historisch Verbürgten und des Legendenhaften in der Tradierung bietet auch zwei Verwendungsmöglichkeiten des Bancbanus-Stoffes in der Literatur an, worauf schon Ernst Joseph Görlich in seinem Aufsatz über Katona und Grillparzer aus dem Jahre 1971 treffend hingewiesen hat: den Banus "als Rächer seiner verletzten Ehre oder als treuen Vasallen seines Königs dar[zu]stellen, als Haupt einer fremdenfeindlichen Verschwörung oder als > treuen Diener seines Herrn «.$^{3}$ Welche dieser beiden Optionen im einzelnen Drama überwiegt, wie sie von Werk zu Werk dosiert werden, dies hängt - wie noch zu zeigen sein wird - weniger mit den ästhetischen als mit den politisch-ideologischen und geschichtsphilosophischen Ansichten ihrer Autoren zusammen; Ansichten, in denen sich Konzepte eines übernational begründeten Gesamtstaates (bei Grillparzer) oder aber eines eigenen, ethnisch definierten Nationalstaates (bei Katona und Marković) erkennen lassen. Zweifellos sind diese Ansichten und Konzepte - davon wird im Weiteren gleichfalls die Rede sein - sehr eng mit der Konstruktion kollektiver und individueller Identitäten im Habsburger Reich verbunden und verweisen zugleich auf eigentümliche politische und kulturelle Asymmetrien des Vielvölkerstaates, vor allem jene zwischen Zentrum und Peripherie, zwischen hegemonialen und subordinierten Völkern und zwischen Herr und Knecht (in unserem Falle doppelt kodiert). Mit Blick auf die Debatte um die Verwendungsmöglichkeit des postkolonialen Begriffsinstrumentariums für Zustände in der Habsburger Monarchie wäre - wie Peter Stachel in einem anderen Kontext bemerkt - hinzuzufügen, dass dabei keineswegs die Rede von nur einem dominanten, zentralistischen »Kolonisierungsdiskurs «, sondern auch von vielfach miteinander verschränkten regionalen »Mikrokolonialismen« sein kann: 
Für die Magyaren [...] war Wien bis 1867 in der Tat ein politisches Zentrum, das als gleichsam ‘äußere Autorität ‘ bekämpft wurde, für die Kroaten als Untertanen der ungarischen Krone hingegen war Budapest dieses als zwangsweise normierend empfundenes Zentrum, demgegenüber Wien ein Gegengewicht darstellte [...]. ${ }^{4}$

Eine vergleichende, nationalismus- wie auch nostalgiekritische Lektüre der drei literarischen Gestaltungen des Bancbanus-Stoffes könnte insofern einen Beitrag zu jener »kritische[n] Revision der Darstellungsformen der k.(u.)k. Monarchie « ${ }^{5}$ bieten, die Clemens Ruthner als ein wichtiges Ziel der postkolonial inspirierten Mittel- und Osteuropaforschung hervorgehoben hat. Dass sich Kulturanalyse und Gesellschaftsanalyse dabei ständig durchdringen und überschneiden werden, liegt auf der Hand.

2.

Grillparzers Stück Der treue Diener seines Herrn wurde vom Publikum Ende der 1820er Jahre begeistert aufgenommen, gehörte später aber zu den weniger gespielten (und auch weniger geschätzten) Werken des österreichischen Klassikers. Im Gegensatz dazu blieb das älteste der drei Bancbanus-Dramen, Katonas Bánk bán, lange unbeachtet und errang seinen (bis heute unumstrittenen) Status des bedeutendsten ungarischen Nationaldramas erst nach der Aufführung im Pester Nationaltheater 1845, zu einer Zeit also, als das sogenannte Reformzeitalter, der ungarische Prozess der nationalen Integration, seinen Höhepunkt erreichte, der Autor selbst aber schon fünfzehn Jahre zuvor verstorben war. ${ }^{6}$

In den Bühnenwerken Grillparzers und Katonas, die eine so unterschiedliche Rezeptionsgeschichte aufweisen, lassen sich große Unterschiede auch in der Gestaltung der Titelgestalt Bancbanus festhalten: Mit dem >treuen Diener seines Herrn ^ des österreichischen Dramatikers, der dem Stück häufig den - wohl ungerechten - Vorwurf des Servilismus einbrachte, hat Katonas Bánk wenig gemeinsam. Ganz im Gegenteil erscheint der Hauptheld in Katonas Drama als ein selbstbewusster ungarischer Aristokrat, der zwar ebenso als ausgesprochen königstreu charakterisiert wird, sich aber nach Verletzung der Rechte der ungarischen Nation und seiner Familienehre - wenn auch unwillkürlich - an die Spitze der Rebellion stellt. Der Widerstand beschränkt sich dabei nicht nur auf den Aufruhr des hohen

4 Stachel: Der koloniale Blick, S. 261.

5 Ruthner: >K.(u.)k. postcolonialı?, S. 103.

6 Vgl. Görlich: Grillparzer und Katona, S. 133f.; Kulcsár Szabó (Hg.): Geschichte der ungarischen Literatur, S. 119-122. Encyclopaedia Britannica bezeichnet Katonas Werk als »the finest Hungarian drama of the 19th century« (Bd. 6, S. 763). 
Adels wegen der Überfremdung des Landes, sondern erhält - wie die Gestalt des aufrechten Bauern Tiborc zeigt - auch eine wichtige soziale Dimension und wird somit zum Anliegen des ganzen Volkes erhoben. Dieser Umstand motiviert zusätzlich die Vermengung der erotischen und der politischen Handlung in Katonas Stück im Sinne einer nationalen Reaktion auf die unrechtmäßigen Eingriffe der Königin Gertrudis, ihres Gefolges und ihres Bruders Otto in ungarische Angelegenheiten:

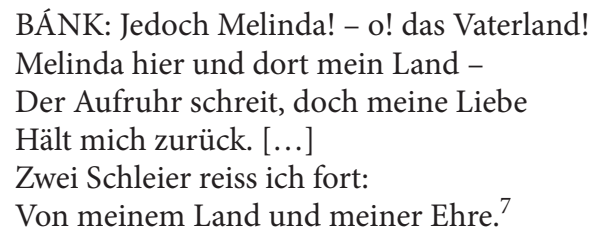

Doch Bánks politischer Aktivismus, der ihn schließlich auch dazu treibt, die Königin zu ermorden, mündet in keinem triumphierenden Patriotismus; aus den Widersprüchen, in die er sich in seinem öffentlichen Handeln sowie im Verhältnis zu seiner Frau Matilda verwickelt, findet der kläglich scheiternde Titelheld keinen Ausweg. Seine tragische Schuld geht nämlich nicht nur aus Gertrudis'- wie sich letztlich zeigt: unnötigen - Tötung hervor; sie trifft ihn auch, weil er, teils aus Unachtsamkeit, teils aus Eifersucht, seine Frau ungeschützt lässt und so ihre Schändung und Ermordung durch fremdländische Gewalttäter mitverschuldet. Folgerichtig erscheint es daher, dass die finale Versöhnung nicht vom völlig niedergedrückten Bánk herbeigeführt werden kann; diese Aufgabe wird an den König delegiert, der sich - von einem siegreichen Kriegszug zurückgekehrt - nicht nur mit dem Tod seiner Gemahlin, sondern mit den ebenso tragischen Folgen des Aufruhrs seiner Vasallen, so auch Bánks, des wohl verlässlichsten unter ihnen, konfrontiert sieht. Als weisem Herrscher, der auch die Schuld der Königin nicht übersieht, leuchtet Andreas ein, dass die Konsolidierung der zutiefst gespaltenen Nation nur durch eine mäßigende Wirkung erreicht werden kann, wobei sich ihm als erster Schritt auf diesem Weg die Begnadigung Bánks anbietet.

Zur Versöhnung sollen symbolisch auch die bevorstehenden Begräbnisfeierlichkeiten für die beiden getöteten Frauen beitragen, deren Leichname gegen Ende des Stückes auf die Szene getragen werden. Und wenngleich man die Schuld der in gefährliche Intrigen verstrickten Gertrudis keineswegs mit jener Matildas vergleichen kann, die der Verführungskunst Ottos doch nicht erliegt und somit ihre moralische Integrität bewahrt, so erscheinen die beiden Frauen - aus einer modernen Perspektive betrachtet - als kol- 
laterale Opfer des männlichen Machtspiels und Begehrens. Ihrer wird auch in der Zusammenführung des erotischen und des politischen Handlungsstrangs gedacht: So wird einerseits Bánk vom König die Erlaubnis erteilt, seine Frau "geziemend $[\mathrm{zu}]$ begraben $«,{ }^{8}$ andererseits bedauert der Monarch in der Schlussreplik, dass die Königin "nicht einig « mit den »edlen Herzen «" der Magyaren sein konnte. Während er seine ungarischen Untertanen - aus Katonas Sicht freilich das ungarische Volk - zugleich zur Überwindung der Zwistigkeiten und zur Versöhnung im nationalpatriotischen Sinne anzuregen sucht (»Magyaren! Ich kenne sie gut - sie lieben mich, / Sind mein! «) ${ }^{10}$ will er auch zum aufgebahrten Leichnam der Königin emporsteigen. Katona, der offenbar die wirkungsvollen, emotionsgeladenen visuellen Effekte des Wiener Volkstheaters kannte und auch in seinem eigenen Stück einzusetzen suchte, lässt den König jedoch plötzlich einen anderen Entschluss fassen: »doch da er an seinen Kindern vorbeikommt, die auf der Treppe stehen, umarmt er sie und kniet nieder «. ${ }^{11}$ Statt seiner schuldbeladenen Gemahlin die letzte Ehre zu erweisen, schließt er seine Kinder, so auch den kleinen Kronprinzen Bela, in die Arme - ein Schlusstableau, das zweifellos viel mehr nationalpatriotisches, weil zukunftsweisendes Potenzial aufweist und zugleich unmissverständlich auch die ideologische Signatur des Dramas zum Ausdruck bringt.

Der Hinweis auf das bühnenwirksame szenische Arrangement stimmt durchaus mit der in der Forschung üblichen Einschätzung überein, Katona habe sich mit Bánk bán von dem in der zeitgenössischen ungarischen Literatur vorherrschenden Klassizismus befreien wollen und dabei namentlich »den geschraubt feinen, antikisierenden Stil « als untauglich empfunden; genauso untauglich erschienen ihm wohl auch die substanzlosen Geschichtsdramen seiner älteren ungarischen Zeitgenossen und deren phrasendreschende Helden: Merkmale, denen er in seinem Stück durch die Verwendung der »Sprache des Volkes $\aleph^{12}$ und durch eine andersartige Heldenkonzeption entgegenwirken wollte. Mit dem Hinweis auf diese Merkmale von Katonas Dramaturgie wird in der Forschung die These belegt, Bánk bán lasse sich als ein früher Text der ungarischen romantischen Literatur kennzeichnen.

Wenig wahrscheinlich ist allerdings Katonas Kenntnis der dramatischen Konzeptionen führender europäischer Romantiker wie Brentano oder Byron, die zeitgleich mit der Entstehung des Bánk bán, also um 1815, an ihren wichtigsten Bühnenwerken schrieben. Von Verbindungslinien zu europäi- 
schen Entwicklungstrends ließe sich wohl in einem anderen Zusammenhang sprechen: im Kontext des Vordringens einer neuen romantischen Kultur und ihrer Kritik an überlieferten ästhetischen Mitteln, unter anderem auch an der traditionellen Heldenkonzeption, denn Katonas Bánk lässt sich durchaus zu jenen typisch romantischen, ambivalenten Helden zählen, welchen Gerhard Schulz drei Merkmale attestiert, »die eng miteinander verbunden sind oder sogar ineinander übergehen «: »ein historisch-gesellschaftlicher, ein psychologischer und ein moralischer « ${ }^{13}$ Im psychologischen Sinne gerät nämlich auch Bánk - wie zahlreiche andere Protagonisten der zwischen 1790 und 1850 entstandenen Werke - in eine melancholische Stimmung, in der sich Gründe und Abgründe seiner Seele abzeichnen. Hinzu kommt seine Bereitschaft, die moralischen Konsequenzen seiner Taten zu tragen und sich mit der physischen und psychischen Vernichtung auseinanderzusetzen. In unserem Zusammenhang scheint jedoch der erstgenannte, der historischgesellschaftliche Aspekt des romantischen Helden noch bedeutender zu sein: Obwohl Bánk besonnen und abwägend zu handeln sucht, obwohl er sich als ein realistischer Politiker vom aufständischen Adel distanziert und auch seine eigenen Emotionen lange beherrschen kann, wird er im Streitdialog mit Gertrudis - in seinem persönlichen und nationalen Ehrgefühl verletzt - plötzlich von einer blinden Leidenschaft erfasst und zum Mord an der Königin getrieben. Dass er durch diese Tat, die ungewollt auch die Ziele der Aufständischen umzusetzen verspricht, keineswegs zum nationalen Befreier werden kann, ist von vornherein offensichtlich; Bánk ist sich denn auch - darin zahlreichen romantischen Helden vergleichbar - seines Fehlverhaltens bewusst und zieht sich deswegen resigniert zurück.

Der seelisch-geistige Niedergang des Titelhelden hat allerdings keine hemmende Wirkung auf die national-patriotische Kodierung des Stückes: Nachdem das Land nun nicht mehr dem fremdländischen Einfluss ausgeliefert ist, steht dem König nichts im Wege, seine eigene Macht zu festigen und dadurch nicht nur die Einheit der Nation zu erzielen, sondern sich darüber hinaus auch dem westlichen Nachbarn gegenüber zu behaupten. Dass sich die von Katona inszenierte Geschichte trotz des mittelalterlichen Settings in einem ausgesprochen modernen Sinne interpretieren lässt, geht schon aus dem folgenden Umstand hervor: das Drama stieß in den 1820er und 1830er Jahren auf ein verhältnismäßig geringes Publikumsinteresse, was sicherlich auch mit Zensurmaßnahmen zusammenhing, ${ }^{14}$ während es in der unmittelbaren Vorrevolutionszeit, auf dem Höhepunkt der ungari-

14 Vgl. Görlich: Grillparzer und Katona, S. 133. 
schen nationalen Bestrebungen, enthusiastisch aufgenommen wurde. Für die mobilisierende Wirkung dürfte vor allem der im Stück thematisierte Aufruhr gegen die fremdländische Usurpation, und in diesem Rahmen namentlich die Tötung der tyrannischen, dazu noch deutschstämmigen Königin durch einen königstreuen, freiheitsliebenden ungarischen Aristokraten entscheidend gewesen sein - jener Aspekt der Handlung also, der symbolisch auch als die angestrebte Befreiung vom deutschösterreichisch dominierten Zentrum des Gesamtstaates verstanden werden konnte. In der historischen Realität wird der ungarische Patriotismus allmählich zum magyarischen Staatsnationalismus, womit auch der Konstruktionsprozess der modernen ungarischen Nation zu seinem Abschluss kommt. Parallel dazu werden auch die national kodierten Bühnenwerke wie Katonas Bánk bán in einem neuen Schlüssel gelesen, wobei vor allem der national bestimmte Aktivismus der Dramenhelden in den Vordergrund gerückt wird. Dass man die Sinnpotenziale solcher kulturellen Konstrukte allerdings nicht nur mit einer Neudefinierung der ungarischen Stellung im Rahmen der Habsburger Monarchie, sondern darüber hinaus auch mit der Begründung eines magyarischen Nationalstaates verband, steht außer Zweifel. ${ }^{15}$

\section{3.}

Einführend wurde schon darauf hingewiesen, wie stark die BancbanusDarstellung in Grillparzers Ein treuer Diener seines Herrn von jener in Katonas Bánk bán abweicht. Dieser Umstand lässt sich in vielerlei Hinsicht - wie auch in den wenigen vergleichenden Untersuchungen zu den beiden Werken hervorgehoben wird ${ }^{16}$ - mit den unterschiedlichen, ja gegensätzlichen Auffassungen der beiden Autoren von dem Verhältnis zwischen dem Monarchen und den Untertanen wie auch von dem übergeordneten staatlichen Rahmen erklären. In diesem Zusammenhang wurde weiter oben die für das ungarische Stück konstitutive antihabsburgische Tendenz erwähnt, die im Text des österreichischen Autors nicht vorliegt: ganz im Gegenteil lässt Grillparzer die Treue des Titelhelden seinem Herrscher gegenüber so unverbrüchlich erscheinen, dass mit diesem Aspekt der Dramenhandlung auch der erwähnte Servilismusvorwurf den Habsburgern gegenüber begründet wird.

Wenngleich sich dieses Urteil bei einer näheren Beschäftigung mit dem Treuen Diener - aber auch mit den beiden anderen, den habsburgischen

15 Vgl. dazu: Rumpler: Österreichische Geschichte 1804-1914, S. 169-214.

16 Außer Görlich vgl auch: Mádl: Grillparzer in Ungarn. 
Komplex thematisierenden historischen Tragödien des Autors (König Ottokars Glück und Ende, Ein Bruderzwist in Habsburg) - entkräften lässt, war es jahrzehntelang Gegenstand bissiger Angriffe auf den sschwarz-gelben< Grillparzer. Argumentiert wurde dabei vor allem mit dem Hinweis auf den bekannten entstehungsgeschichtlichen Umstand, ${ }^{17}$ dass Grillparzer vom Wiener Hof dazu aufgefordert wurde, anlässlich der Krönung der Gemahlin des Kaisers Franz I. zur Königin von Ungarn ein Festspiel aus der ungarischen Geschichte zu schreiben. Desgleichen wurde dem Autor vorgeworfen, der Kaiser sei von dem Stück so begeistert gewesen, dass er es nur für sich hätte haben wollen. Obwohl es sich dabei nur um Halbwahrheiten handelte, obwohl Grillparzer wegen des Treuen Dieners nur frustrierende Erfahrungen mit dem Hof machte, und obwohl das Stück bald nach der Uraufführung vom Spielplan verschwand, wurde dem Autor wegen diesem Stück, aber auch aus weiteren Gründen, eine unkritische Glorifizierung der österreichischen Dynastie angelastet. Im deutschsprachigen Raum geschah dies namentlich im deutschnational gesinnten Lager der Kritiker, während bei nichtdeutschsprachigen Völkern der Monarchie diesbezügliche Vorwürfe vor allem von antihabsburgisch orientierten Intellektuellen erhoben wurden.

Dass diese Urteile nicht auf ästhetischen, sondern auf politischen Argumenten fußen und dass sich dabei - wie noch zu zeigen sein wird - vor allem entgegengesetzte Loyalitätsoptionen dem habsburgischen Zentrum gegenüber als ausschlaggebend erweisen, stand schon für die Zeitgenossen außer Zweifel. Grillparzer, ein überzeugter Josephiner, betrachtete die Donaumonarchie als den für die Völker Mitteleuropas angemessenen staatlichen Rahmen und sah ihre Aufrechterhaltung in der dominanten Position des deutschsprachigen Kerns gewährleistet. Folgerichtig erscheint es daher, dass er mit den Zuständen im Vielvölkerstaat zutiefst unzufrieden war und dies auch oft zum Ausdruck brachte, wobei sich seine Kritik vor allem auf zwei Bereiche bezog: auf die seiner Meinung nach völlig unnötige Unterdrückung des Geisteslebens durch Zensur- und Polizeimaßnahmen einerseits, und andererseits auf die Unfähigkeit des habsburgischen Staates, den Emanzipationsbestrebungen nichtdeutschsprachiger Völker mit einer konsistenten Politik zu begegnen. Die schmerzliche Erkenntnis, dass zwischen dem von ihm erhofften, idealen Österreich und der erbärmlichen Realität des Metternich'schen Systems eine unüberwindbare Kluft besteht, gehört zu den zentralen Erfahrungen des Dichters und findet starken Niederschlag in seinen zahlreichen Werken, so auch in Ein treuer Diener seines Herrn.

17 Vgl. Franz Grillparzer: Sämtliche Werke, Bd. I, S. 1318; vgl. auch Görlich: Grillparzer und Katona, S. 129. 
Obwohl sich Grillparzer in seinen Reflexionen über zentripetale Tendenzen bei anderssprachigen Völkern der Monarchie mancherorts auch zu Behauptungen verleiten ließ, die von einem späteren Gesichtspunkt aus kaum verständlich sind, wie beispielsweise jener, die ungarische Sprache habe keine Zukunft, ${ }^{18}$ so stehen auf der anderen Seite Grillparzers sicherlich bedeutendere Bemühungen um dramatische Transponierungen von Stoffen aus der tschechischen und ungarischen Geschichte. Die für das gesamtösterreichische Narrativ zentrale Idee einer `Einheit in Vielheit، und einer damit verbundenen engeren Verbindung verschiedener Völker der Monarchie suchte Grillparzer dabei »mit historischen Argumenten, die ein notwendiges Zusammengehen mit Beispielen aus der Vergangenheit demonstrieren sollten, zu untermauern ${ }^{19}{ }^{19}$

Das Anliegen des Dichters, das erwünschte Zusammenwachsen der Völker in seinem `habsburgischen Dramenzyklus zu thematisieren und damit zum Abbau von nationalen Spannungen beizutragen, schlug jedoch sowohl bei den Tschechen als auch bei den Ungarn fehl; nicht viel anders verlief die Rezeption dieser Werke Grillparzers auch bei anderen nichtdeutschsprachigen Völkern des Habsburgerreiches. ${ }^{20}$ Das Prinzip des Nationalstaates und das Prinzip des überethnischen Reiches standen sowohl im Vormärz als auch danach so unversöhnlich einander gegenüber, dass ein Transfer national bzw. übernational kodierter Texte innerhalb der Monarchie häufig auf unüberwindliche Hindernisse stieß: So kann es nicht verwundern, dass Grillparzers Darstellung des Königs Ottokar den Tschechen missfiel, genauso wie den Ungarn seine Bancbanus-Bearbeitung fremd blieb und somit weder ins Ungarische übersetzt noch auf einer ungarischsprachigen Bühne aufgeführt wurde.

Wie Antal Mádl bemerkt, wirkte sich auf Grillparzers Bancbanus-Stück auch »die `ungarische Konkurrenz` durch József Katonas Drama nachteilig « aus: ${ }^{21} »$ Es war früher entstanden und gestaltete den Banus nicht als streuen Diener seines Herrn`, sondern als einen betont selbstbewussten, auf seine Würde achtenden Anführer der ungarischen Aristokratie und als Verteidiger der Dorfarmut. $\aleph^{22}$ Indem Katonas Stück, so der ungarische Germanist, »zum Nationaldrama« wurde,

20 Besonders drastisch im kroatischen Fall: vgl. Bobinac: Zwischen Übernahme und Ablehnung, S. 141-177.

21 Anzumerken wäre allerdings, dass Grillparzer Katonas Stück vor der Niederschrift seines Treuen Dieners in den 1820er Jahren nicht kannte (Görlich: Grillparzer und Katona, S. 130); Mádls Formulierung von der `Konkurrenz bezieht sich wohl auf die Zeit nach 1840, als der bis dahin vergessene Bánk bán in Ungarn zum Nationaldrama avancierte.

22

Mádl: Grillparzer in Ungarn, S. 97. 
war [es] geeignet, mit dem Haß gegen das Metternichsche System gleichzeitig auch den Widerwillen gegen das Haus Habsburg zum Ausdruck zu bringen. Unter diesen Umständen konnte Grillparzer [...] bei einem einigermaßen politisch informierten Publikum nur auf Ablehnung stoßen. ${ }^{23}$

Die Frage, ob sich Ein treuer Diener seines Herrn tatsächlich als Habsburg-Apologie lesen lässt oder eben nicht, hängt offensichtlich mit der politischen Einstellung des Betrachters zusammen und wurde in der älteren Publizistik und Forschung unterschiedlich beantwortet. Zu diesem früher heftig umstrittenen Aspekt der Grillparzer-Rezeption überwiegen in der neueren Zeit jedoch ausgewogene Urteile, wobei nicht selten der politische Hintergrund weitgehend ausgespart wird. ${ }^{24}$ Für Claudio Magris gehört das Bancbanus-Stück zu den frühen Belegen für die These von der konstitutiven Bedeutung des habsburgischen Mythos für die österreichische Literatur: Unter den spezifisch habsburgischen Themen hebt der italienische Germanist insbesondere die Treue zum anvertrauten Amt, die Bewahrung der Autorität des Herrschers, die Aufrechterhaltung der Ordnung, die pedantische Einhaltung von Gewohnheiten und Routinen bei gleichzeitiger Zärtlichkeit und Liebe im Umgang mit den Nächsten hervor. All diese Eigenschaften, diese »Umdämmung des drängenden Lebens und des zerstörenden Laufs von Werden und Verändern « - so Magris - »wird in der Gestalt des Bancbanus beispielhaft charakterisiert und geht als Erbe in die gesamte spätere habsburgische Literatur ein «. ${ }^{25}$ Und wenige Zeilen danach erklärt er Bancbanus zum »Stammvater einer Reihe von Gestalten der österreichischen Literatur «. ${ }^{26} 27$

Den grundlegenden Eigenschaften der Bancbanus-Figur wie Treue zum König und Aufrechterhaltung der staatlichen Ordnung begegnet man offensichtlich sowohl bei Katonas als auch bei Grillparzers Titelhelden; letzterer lässt sich im Gegensatz zu Katonas Bánk jedoch weder zu Eifersucht noch zu heftigen Reaktionen verleiten, sei es zu national gefärbten Beschimpfungen, sei es zu einem wie auch immer berechtigten Mord. Auch in den äußersten existenziellen Situationen, mit denen er sich während der Abwesenheit des Königs als Reichsverweser tragen muss, bleibt Grillpar-

23 Ebd.

24 Vgl. z.B. Politzer: Grillparzer, S. 185ff. sowie Škreb: Grillparzer, S. $150 \mathrm{ff}$.

25 Magris: Der habsburgische Mythos, S. 109.

26 Ebd., S. 110.

27 Verwirrend ist allerdings Magris' Behauptung, Grillparzers Bancbanus gemahne »an den großen Diener und Kanzler der Monarchie, an Metternich« (ebd., S. 109), hat der Dichter doch immer auf Distanz zum langjährigen Reichskanzler gestanden und die Methoden seiner Politik kritisiert. Dass er - wie Metternich - positiv zur Idee der Habsburgermonarchie eingestellt war, steht auf einem anderen Blatt. 
zers Bancbanus - wie Heinz Politzer bemerkt - wie ein "steinerner Gast in einer Welt voll Leidenschaften $«{ }^{28}$ So nimmt er den Verlust seiner Frau hin, rettet seinen Nebenbuhler Otto, den Bruder der Königin, schlägt den Aufstand nieder und legt zum Schluss die Herrschaft wieder in die Hände des zurückgekehrten Königs. Die Aufforderung seines Bruders Simon und anderer rebellierender Aristokraten, sich an dem Aufruhr zu beteiligen, lehnt der Banus entschieden ab:

Aufrührer! Ich mit euch? - Ich bin der Mann des Friedens,

Der Hüter ich der Ruh - Mich hat mein König

Geordnet, seinen Frieden hier zu wahren;

Ich in den Bürgerkrieg mit euch?

Fluch, Bürgerkrieg! Fluch dir vor allen Flüchen! ${ }^{29}$

Nicht nur der Titelheld, auch andere Figuren in dem für den BancbanusStoff bestimmenden Fünfeck weichen in vielerlei Hinsicht von Katonas Charakterisierung ab, ein Umstand, der zweifellos mit Grillparzers raffinierter, an der Weimarer Klassik orientierter Dramaturgie, insbesondere mit seiner verfeinerten Handlungsmotivierung zusammenhängt. So werden im Treuen Diener weder die Königin, bei Katona wie erwähnt als herrschsüchtig und intrigant gekennzeichnet, noch ihr Bruder Otto, im ungarischen Stück ein Wüstling, der vor Mord und Vergewaltigung nicht zurückschreckt, als eindeutige Bösewichte, sondern eher als ambivalente Gestalten dargestellt; dementsprechend fällt auch ihr Schicksal innerhalb der Dramenhandlung nicht so drastisch aus. Gertrude wird nicht von Bancbanus, sondern von einem Aufständischen ermordet, und zwar versehentlich; ihr Bruder wiederum, der bei Katona nach begangenen Verbrechen flieht, wird bei Grillparzer des Landes verwiesen, nachdem er seine Schuld durch die Rettung des Kronprinzen beglichen hat. In Verbindung mit der Faszination, die für sie vom jungen und attraktiven Otto ausgeht, wird auch Bancbanus' Gemahlin (bei Grillparzer Erny genannt) ambivalent charakterisiert, da ihr Selbstmord vor allem durch die Gefahr motiviert wird, Ottos Verführungskünsten zu unterliegen. Aber weder ihr zunächst irrtümlicherweise als Mord gedeuteter Tod, der den Aristokraten als Anlass zu ihrer Rebellion dient, noch der Tod der Königin können im Gegensatz zu Katonas Drama im nationalen Schlüssel gedeutet werden.

Nicht weniger ambivalent wirkt schließlich auch König Andreas, der bei Grillparzer am Anfang und am Ende des Stückes erscheint, in einer Art dramatischer Umrahmung: Trotz seines Charismas trägt er die eigentliche Schuld am tragischen Geschehen, da er zunächst auf das Beharren seiner Frau die Ernennung Ottos zu ihrem Mitherrscher während seiner Anwesen- 
heit erwägt, später aber dem treuen Bancbanus diese Rolle aufzwingt, ohne daran zu denken, wie das Land unter diesem störrischen Paar zu regieren sein wird. ${ }^{30}$ Als Bancbanus' Regiment wieder auf den zurückgekehrten König übertragen wird, ist das Land schon befriedet, der Aufruhr niedergeschlagen und der streue Diener mit Otto und dem Kronprinzen Bela zu Füßen des Königs. Die Wiederkehr der Harmonie im zerrütteten Reich ermöglicht dem König, seinen Teil der Verantwortung zu übernehmen; Bancbanus wiederum kann sich erst jetzt - all seiner Ämter entledigt - auf sein Schloss zurückziehen und den Tod seiner geliebten Frau betrauern: $[$ [...] dem glücklosen Stellvertreter bleibt [...] die Aufgabe, Treue zu wahren, unter Hintansetzung aller [...] persönlichen Interessen «. ${ }^{31}$

Es wurde darauf hingewiesen, wie Katona gegen Ende seines Dramas die Perspektive vom niedergeschlagenen Bánk auf den König verschiebt und dabei dessen unantastbar erscheinende Macht sowie die wiedererreichte nationale Eintracht in einem effektvollen Schlusstableau zelebrieren lässt. Im Gegensatz dazu bleibt Grillparzers Fokus auch in der letzten Szene auf dem Titelhelden, dessen letzte, zugleich das Stück abschließende Replik sich zuerst an den König, dann auch an dessen Sohn und Nachfolger wendet. Nicht zu übersehen ist, dass nicht der König - wie bei Katona -, sondern Bancbanus vor dem Kronprinzen Bela kniet und dabei - aus der Perspektive eines alten und treuen Dieners der Dynastie sprechend - dem aktuellen und dem zukünftigen Monarchen zu einer humanen, gerechten und gesetzmäßigen Herrschaftspraxis rät.

Vom Nationalen aber, dem man bei Katona auf Schritt und Tritt begegnet, ist an dieser Stelle - wie auch im ganzen Drama - keine Rede. Nicht einmal beim Aufstand des Adels dient die Schreckensherrschaft der Fremden als Anlass; genannt wird ausschließlich die Ermordung Ernys durch Otto - eine Anschuldigung, die sich jedoch als unberechtigt erweist. Dass Grillparzer eine tiefe Aversion gegenüber dem deutschen wie auch den Nationalismen anderer europäischer Nationen hegte, ist wohl bekannt und stand in der Forschung häufig zur Diskussion. Hingewiesen wurde häufig auch auf sein Interesse an der Geschichte des Habsburgerreiches, das unter anderem in seinen historischen Dramen zum Ausdruck kommt und aufs Engste mit seiner Vorstellung von Österreich als einem multinationalen Reich verbunden ist. ${ }^{32}$ Folgerichtig erscheint es daher, dass sich die Treuebekenntnisse zur Dynastie und zum Staat, die sich in Bancbanus' Aussagen manifestieren, in den Kontext des Hauses Habsburg und der

32 Vgl. neuerdings z.B.: Lorenz: Grillparzer's Attitude toward the State, the Nation, and Nationalism. 
Donaumonarchie bringen lassen. Obwohl es selbstverständlich falsch wäre, die Aussagen einer fiktiven Figur dem Autor in den Mund zu legen, der sich bekanntlich bei vielen Anlässen kritisch über die Herrschaftspraxis der Habsburger geäußert hatte, so steht doch außer Zweifel, dass sich im Treuen Diener wie auch in Grillparzers anderen fiktionalen und nichtfiktionalen Texten die Überzeugung niederschlägt, der österreichische Gesamtstaat mit seinem deutschsprachigen Zentrum sei den herbeigesehnten nationalstaatlichen Entwürfen einzelner Völker der Monarchie überlegen. Interessant wäre es, Grillparzers Österreich-Vision genauer in Verbindung mit dem habsburgisch-zentralistischen, von den nichtdeutschsprachigen Nationen als hegemonial aufgefassten Narrativ zu untersuchen; einem Narrativ, das Catarina Martins im Kontext der postkolonialen Theorie folgendermaßen beschreibt:

Wie Said für die überseeischen Kolonialmächte feststellt, ist in der Habsburger Monarchie die Erzählung des Imperialismus auch Teil, Fortsetzung und Konsolidierung der erzählenden Konstruktion der Nation als >imagined community< (Anderson). In diesem Falle geschieht dies aber durch den verstrickten Umweg, der es erlaubt, mit dem Instrument des Multikulturalitätsdiskurses den Nationalismus als zentrifugale und zersetzende Dynamik in eine zentripetale, zentralisierende, staatserhaltende Bewegung zu transformieren. ${ }^{33}$

\section{4.}

Unter den kroatischen Intellektuellen des 19. Jahrhunderts, die die Ablehnung von Grillparzers Werk politisch-ideologisch begründeten, ist insbesondere der Schriftsteller und Philosoph Franjo Marković (1845-1914) hervorzuheben. Bevor er um die Mitte der 1870er Jahre eine Professur an der neubegründeten Universität Zagreb antrat und sich in der darauffolgenden Zeit als Theoretiker der Ästhetik profilierte, hatte Marković bereits das Gros seines literarischen Werks verfasst, das neben Balladen und Verserzählungen auch einige historische Trauerspiele, so auch ein Bancbanus-Drama enthält. In diese Zeit fallen auch seine Doktorstudien bei dem Philosophieprofessor Robert Zimmermann in Wien, wo er vor seiner Promotion 1872 die Gelegenheit hatte, die Feierlichkeiten zu Grillparzers achtzigstem Geburtstag 1871 und daraufhin auch jene zum Tode des Dichters 1872 aus nächster Nähe beobachten zu können.

Doch weder das Werk des Dichters noch der Pomp, mit dem Grillparzer in Österreich zum Schluss seines Lebens gefeiert wurde, beeindruckten- wie es scheint - den Dissertanten aus Kroatien. In einem Aufsatz, den er in der 
von ihm redigierten literarischen Zeitschrift»Vienac« 1870 veröffentlichte, erklärt Marković Grillparzer zu einem »Dramatiker des zweiten oder gar des dritten Ranges ${ }^{34}$ und setzt ihn dabei mit älteren österreichischen Theaterautoren wie Ayrenhoff und Collin gleich, die ebenso nichts anderes als Lobgesänge auf die österreichische Dynastie geschrieben hätten: Grillparzer - für Marković im Grunde ein politischer Dramatiker - habe in seinem Drama König Ottokars Glück und Ende eine »Apotheose des Hauses Habsburg ${ }^{35}$ vorgelegt und dadurch die tschechische Geschichte völlig entstellt gezeigt, ein Umstand, auf den - so Marković, aber auch viele andere Grillparzer-Kritiker davor und danach - Tschechen und andere Slawen mit Recht empört reagiert hätten. ${ }^{36}$

Grillparzers »unterwürfige Treue zum Herrscher « in Ein treuer Diener seines Herrn wurde, so Marković, sogar "von deutschen Kritikern als >hündisch bezeichnet $« .{ }^{37}$ Noch mehr zeigt er sich darüber irritiert, dass Grillparzer in diesem Stück - wie auch andere deutsche und ungarische Dramatiker vor ihm - Persönlichkeiten aus der kroatischen Geschichte ausschlachte, denn der Held des Dramas, Bancbanus, sei eigentlich - so Marković im Widerspruch zu den Angaben in den historischen Quellen der kroatische Aristokrat Benko Bot gewesen. Auch darin erweise sich »das politische, den Zentralismus und Absolutismus verteidigende deutschösterreichische Drama [...] als ein Sklave der einheimischen politischen Lüge«; wie andere Produkte dieser Art sei auch Ein treuer Diener seines Herrn "nur ein vergänglicher Versuch ohne jeglichen ästhetischen Wert, genauso wie der Zweck, dem es als Mittel diente, ein wertloser Versuch war «. ${ }^{38}$

Als ein wichtiges Anliegen seiner eigenen schriftstellerischen Tätigkeit wird Marković die Begründung eines neuartigen historischen Dramas in Kroatien ansehen - selbstverständlich in Einklang mit der kroatischen Staatsidee. Bereits 1872, zwei Jahre nach dem Erscheinen seines GrillparzerAufsatzes und ein halbes Jahrhundert nach der Entstehung der BancbanusDramen von Grillparzer und Katona, präsentiert er seine eigene Deutung des Stoffes in dem Trauerspiel Benko Bot (E, D 1872, UA 1899): Als kroatischer Banus unterliegt der Titelheld, und mit ihm auch sein Vaterland, dem vereinigten ungarisch-deutschen Gegner. Dieses sowie andere Dramen und Epen Markovićs sind einer klassizistisch-historistischen Ästhetik verpflichtet und thematisieren vor allem kroatisch-ungarische Konflikte im Spätmittelalter, 
die unmissverständlich auf die Gegenwart im Vielvölkerstaat ÖsterreichUngarn und namentlich auf die Eingriffe der beiden dominanten Nationen in die Geschicke der slawischen Völker anspielen.

Folgerichtig erscheint es, dass sich in Markovićs literarischer Programmatik - und insbesondere in seinem Bancbanus-Drama - klare Bezüge zu seiner philosophischen Tätigkeit erblicken lassen: Vom »Herbart'schen schulphilosophischen System « ausgehend, sah er ein zentrales Anliegen darin, »die Autonomie der kroatischen Nationalphilosophie gegenüber den dominierenden philosophischen Strömungen der großen europäischen Völker zu sichern « und »insbesondere die kroatische Philosophie vor dem germanischen Einfluß [...] zu schützen ${ }^{39}{ }^{39}$ In diesem Sinne ist auch seine »These über die nationalen Ästhetiken« zu verstehen: denn laut Marković verfügen verschiedene Völker über »ihre festgelegten ästhetischen Regeln, ihren ästhetischen Geschmack.< [...] Er äußert `Zweifel [darüber], daß sich der Philosoph als Ästhetiker dem Geschmack seines Zeitalters, seines Volkes entziehen könnte ‘. ${ }^{40}$

Diesen Ansichten Markovićs, die sich im Sinne der theoretischen Ansätze des Postkolonialismus als Protest gegen den kulturellen Imperialismus hegemonialer gegenüber subordinierten Nationen der Monarchie deuten lassen, entspricht auch der Aktivismus, der zu den wesentlichen Charaktereigenschaften seines Protagonisten Bancbanus alias Benko Bot gehört: Er ist zwar durch Königstreue gekennzeichnet, engagiert sich aber auch - nicht unähnlich dem Titelhelden Katonas, im Gegensatz aber zu jenem Grillparzers - entschieden für die nationale Sache. Hinzuzufügen wäre, dass Benko wie auch andere Hauptfiguren Markovićs ethnisch eindeutig bestimmt ist, wobei neben dem Banus auch seine junge Gattin, die hier Jelina heißt, als Kroatin auftritt. Die Königin Gertrud/a und ihr Bruder, der den Namen Hinko (=Heinrich) trägt, erscheinen auch bei Marković als Deutsche, so dass im Protagonisten-Fünfeck nur der König Andrija (Andreas) zur ungarischen Nation gehört.

Mit nationalem Vorzeichen versehen sind auch erotische Rivalität und politische Ranküne, die im kroatischen Bancbanus-Stück - darin den beiden anderen verwandt - als zentrale Triebfeder der Dramenhandlung erscheinen, selbstverständlich mit anderen Akzentsetzungen: Wie bei Katona erscheint auch hier die Königin als eine skrupellose, grausame Intrigantin, die vor nichts zurückschreckt, um das innige Vertrauensverhältnis des Königs zu seinem Statthalter und Berater Benko zu zerstören. Ihrem Bruder Hinko gelingt es jedoch bei Marković - im Gegensatz zu Katonas Otto -, 
dem unheilvollen Einfluss der Schwester zu widerstehen: Er wird als ein weltfremder Dichter und darüber hinaus als ein einfühlsamer, opferbereiter Mensch dargestellt, der Jelina zwar in rein platonischem Sinne zärtlich liebt, gleichermaßen aber auch ihren Mann Benko aufrichtig verehrt. Von Gertruda wird - als Höhepunkt ihrer Kabale - ein heimliches Treffen zwischen Hinko und Jelina arrangiert, zu dem sie auch Benko schickt, der aber die List der Königin durchschaut und sie in einem Anfall von Wut umbringt. Auch Jelina findet ein tragisches Ende: sie begeht Selbstmord, als sie und Hinko von ihrem Mann überrascht werden; Benko rächt sich jedoch nicht an seinem Nebenbuhler, sondern begibt sich mit Jelinas Leichnam nach Kroatien, während der erschütterte Hinko den Schwestermord gesteht und zu Tode verurteilt wird. Als Benko davon erfährt - die kroatischen Aristokraten, wegen Jelinas Tod empört, planen inzwischen einen Aufstand gegen die fremdländische Macht -, eilt er nach Ungarn zurück und bekennt dem König, die Königin habe er, und nicht ihr Bruder, ermordet. Unmittelbar darauf erscheinen auch die Aufständischen aus Kroatien, ihre Erhebung wird jedoch von königlichen Truppen niedergeschlagen, und die Anführer, zusammen mit Benko, enden auf dem Schafott.

Im Unterschied zum subtil verfahrenden Grillparzer, aber auch zu dem weniger zimperlichen Katona, deren Stücke versöhnend ausklingen, lässt Marković sein Bancbanus-Drama mit einem verzweifelten Aufruhr empörter Kroaten und der daraufhin erfolgten Hinrichtung der Rebellen samt dem Titelhelden, somit in einem kämpferischen, ja märtyrologischen Ton enden. Die Darstellung des desperaten Aktivismus in Benko Bot hängt zweifellos auch mit der prekären politischen Lage in Kroatien Ende der 1860er und Anfang der 1870er Jahre zusammen - einer Zeit, in der sich alle politischen Lager im Protest gegen den kroatisch-ungarischen Ausgleich von 1868 vereinigten und dieses »Knebelungswerk $\aleph^{41}$ als aufgezwungen und für die kroatische Seite stark diskriminierend ablehnten. $\mathrm{Zu}$ den radikalen Aktionen nationalistischer Aktivisten aus diesem Anlass gehörte ein Aufstand in der kroatischen Militärgrenze Ende 1871, gegen den das Militär besonders brutal verfuhr und in dem zahlreiche Aufständische getötet wurden, unter anderen auch Eugen Kvaternik, ein bekanntes Mitglied der kroatischen Staatsrechtspartei, der in der Folgezeit ins kollektive Gedächtnis als Märtyrer im Kampf gegen die habsburgische Gewalt eingegangen war. ${ }^{42}$ Bedenkt man, dass auch Markovićs Benko Bot einen kroatischen Aufstand gegen die ungarisch-deutsche Zentralmacht thematisiert, so kann es nicht

41 Rumpler: Österreichische Geschichte 1804-1914, S. 443.

42 Vgl. Goldstein: Hrvatska povijest, S. 189-191 sowie Rumpler: Österreichische Geschichte 1804-1914, S. 441-445. 
verwundern, dass das Stück zwar in Druck erscheinen, doch seiner politischen Brisanz wegen im Theater vorerst nicht gespielt werden konnte. ${ }^{43}$

Wenngleich sich die Entstehungszeit des Stückes von den Vormärzverhältnissen, mit denen sich die Bühnenwerke von Katona und Grillparzer konfrontiert sahen, in vielerlei Hinsicht unterscheidet, so muss zweifelsohne auch Benko Bot im Kontext des im kroatischen Fall noch immer nicht abgeschlossenen `nation-building«-Prozesses betrachtet werden. Vor diesem Hintergrund ließe sich Markovićs Drama auch als eine frühe literarische Reaktion auf die Neuauflage der politischen und kulturellen Asymmetrien lesen, auf eine Konstellation, die sich nach der Kompromissbildung zwischen den beiden mächtigsten Nationen des Habsburgerreiches im österreichischungarischen Ausgleich 1867 abzuzeichnen und sich in vielerlei Hinsicht zu Schaden anderer Nationen zu konsolidieren begann. Während Katona fünfzig Jahre zuvor am Ende seines Stückes einen resignierten, ja zerschlagenen Banus neben einem aktivistisch eingestellten König präsentiert, wird das Dramenende bei Marković im entgegengesetzten Sinne gestaltet: Die Schlussreplik gehört nämlich dem Banus, der - bevor man ihn zur Hinrichtung abführt - dem kroatischen Volk eine glorreiche Wiedererstehung ankündigt, dem plötzlich mutlos scheinenden König hingegen eine düstere Prophezeiung entgegen wirft: Andreas' Land - so Benkos Worte - solle bald von »blutrünstigen Tieren aus dem mongolischen Sande« vernichtet werden; im Gegensatz dazu werden die hingerichteten Kroaten,

[...] diese Opfer, mein Geschlecht,

ihre gebrochenen Glieder aus den Gräbern erheben

und auferstehen wird auch das kroatische Volk.

[...] Ich soll jetzt das Schafott besteigen. Wenn für mein Volk

bessere Zeiten kommen, wird man sich meiner erinnern. ${ }^{44}$

Es wurde darauf hingewiesen, wie sich die mobilisierende Wirkung von Katonas Bánk ban vor allem aus dem Aufruhr gegen die fremdländische Usurpation speist, einem Handlungsaspekt, der symbolisch auch als Befreiung vom deutschösterreichisch beherrschten Zentrum der Monarchie verstanden werden konnte. Als habsburgisch-zentralistisch wurde

43 Die Uraufführung des Stückes im Zagreber Kroatischen Nationaltheater im Jahr 1899, also 27 Jahre nach der Entstehung, wurde vom Publikum allerdings kaum beachtet. Im Feuilleton wurde zwar sein patriotisches Potenzial gelobt, das Drama selbst aber nur als ein bemerkenswertes Jugendwerk eines anerkannten Philosophen und Universitätsprofessors zur Kenntnis genommen. In den Besprechungen der Aufführung wird auch auf die ein Vierteljahrhundert zurückliegenden Zensurmaßnahmen gegen das Bühnenwerk hingewiesen (vgl. "Narodne novine« 4.9.1899; »Obzor« 4.9.1899; »Agramer Zeitung« 4.9.1899; »Agramer Tagblatt« 4.9.1899).

44 Franjo Marković: Benko Bot, S. 833 (übersetzt von M. B.). 
hingegen Grillparzers Vision eines gesamtstaatlichen Rahmens in seinem Drama Ein treuer Diener seines Herrn vorgestellt: aufgebaut im Sinne einer supranationalen >Einheit in der Vielheit‘, freilich vom deutschsprachigen Kern dominiert. Dieser Vision entspricht allerdings nicht die Lage Kroatiens, das sich im Abhängigkeitsverhältnis zu zwei andersethnischen Herrschaftszentren, zu Wien und zu Budapest befindet, ein Umstand, der auch Markovićs krisenhafte Wahrnehmung der dualistischen Neuordnung der Monarchie in Benko Bot bestimmt. Der aussichtlose Widerstandskampf und das anschließende Martyrium kroatischer Rebellen mit dem Banus an der Spitze bieten sich nämlich als ein symbolisches Handeln dar, dessen semantische Potenziale in der politischen Realität der Donaumonarchie auf eine gleichzeitige Bekämpfung der deutschösterreichischen und der ungarischen Hegemonialstellung auf der einen sowie auf angestrengte Bemühungen um die Erreichung eines föderalistisch reformierten gesamtstaatlichen oder aber eines eigenen nationalstaatlichen Rahmens zu suchen wären.

Die drei vorgestellten Fassungen des Bancbanus-Stoffes stellen daher nicht nur drei kulturelle Konstrukte dar, in denen sich die für den Habsburger Vielvölkerstaat charakteristischen kulturellen Differenzen artikulieren. Die Bühnenwerke von Katona, Grillparzer und Marković weisen gleichermaßen darauf hin, dass »die Frage der kulturellen Differenz untrennbar mit der Frage der politischen und gesellschaftlichen Macht verbunden ist ${ }^{45}$

\section{Literaturverzeichnis}

Bobinac, Marijan: Zwischen Übernahme und Ablehnung. Aufsätze zur Rezeption deutschsprachiger Dramatiker im kroatischen Theater. Wrocław, Dresden: ATUT-Neisse 2008, S. 141-177.

Csire, Márta u.a. (Hgg.): Ein Land mit Eigenschaften. Sprache, Literatur und Kultur in Ungarn in transnationalen Kontexten. Zentraleuropäische Studien für Andrea Seidler. Wien: Praesens 2015.

Goldstein, Ivo: Hrvatska povijest. Zagreb: Novi Liber 2003.

Görlich, Ernst Joseph: Grillparzer und Katona. Bánk-Bán in ungarischer und österreichischer Sicht. »Ungarn-Jahrbuch « 3 (1971), S. 123-134.

Grillparzer, Franz: Sämtliche Werke. Hgg. Peter Frank, Karl Pörnbacher. Bd. I. München: Hanser 1960.

Hrvatski biografski leksikon, Bd. I. Zagreb: JLZ 1983.

Hyršlová, Květa: Grillparzer und Böhmen. Versuch einer Neubewertung. In: Grillparzer oder Die Wirklichkeit der Wirklichkeit. Hgg. Bernard Denscher, Walter Obermaier. Wien: Wiener Stadt- und Landesbibliothek 1991, S. 90-95. 
Katona, József: Bánk bán. Drama in fünf Akten. Übertragung von Jenő Mohácsi. Budapest: Corvina 1955.

Klaić, Vjekoslav: Povijest Hrvata. Bd. I. Zagreb: Nakladni zavod Matice Hrvatske 1982.

Kulcsár Szabó, Ernő (Hg.): Geschichte der ungarischen Literatur: Eine historisch-poetologische Darstellung. Berlin, Boston: De Gruyter 2013.

Lorenz, Dagmar C. G.: Grillparzer's Attitude toward the State, the Nation, and Nationalism. In: Aneignungen, Entfremdungen. The Austrian Playwright Franz Grillparzer (1791-1872). Hgg. Mariann Henn, Clemens Ruthner, Raleigh Whitinger. New York u.a.: Peter Lang 2007, S. 1-20.

Mádl, Antal: Grillparzer in Ungarn. In: Grillparzer oder Die Wirklichkeit der Wirklichkeit. Hgg. Bernard Denscher, Walter Obermaier. Wien: Wiener Stadt- und Landesbibliothek 1991, S. 96-104.

Magris, Claudio: Der habsburgische Mythos in der österreichischen Literatur. Übersetzt von Madeleine von Pásztory. 2. Aufl. Salzburg: Otto Müller 1988.

Marković, Franjo: Benko Bot. »Vienac« 49 (1872), S. 773-777; 50 (1872), S. 789-793; 51 (1872), S. 809-812; 52 (1872), S. 825-833.

Marković, Franjo: Narodno kazalište. »Vienac« 23 (1870), S. 372-374.

Martins, Catarina: Imperialismus des Geistes. Fiktionen der Totalität des Ichs in der österreichischen Moderne. In: Kakanien revisited. <http://www.kakanien.ac.at/beitr/postcol/ CMartins1.pdf> (Zugriff 27.3.2014).

Niedermüller, Peter: Der Mythos des Unterschieds: Vom Multikulturalismus zur Hybridität. In: Habsburg postcolonial. Machtstrukturen und kollektives Gedächtnis. Hgg. Johannes Feichtinger, Ursula Prutsch, Moritz Csáky. Innsbruck u.a.: Studien-Verlag 2003, S. 69-80.

Politzer, Heinz: Grillparzer oder das abgründige Biedermeier. Wien: Molden 1972.

Rumpler, Helmut: Österreichische Geschichte 1804-1914. Eine Chance für Mitteleuropa. Bürgerliche Emanzipation und Staatsverfall in der Habsburgermonarchie. Wien: Ueberreuter 1997.

Ruthner, Clemens: `K.(u.)k. postcoloniale? Für eine neue Lesart der österreichischen (und benachbarten) Literatur/en. In: Kakanien revisited. Das Eigene und das Fremde (in) der österreichisch-ungarischen Monarchie. Hgg. Wolfgang Müller-Funk, Peter Plener, Clemens Ruthner. Tübingen, Basel: Francke 2002, S. 93-103.

Schulz, Gerhard: Romantik. Geschichte und Begriff. München: Beck 2002.

Stachel, Peter: Der koloniale Blick auf Bosnien-Herzegowina in der ethnographischen Popularliteratur der Habsburgermonarchie. In: Habsburg postcolonial. Machtstrukturen und kollektives Gedächtnis. Hgg. Johannes Feichtinger, Ursula Prutsch, Moritz Csáky. Innsbruck u.a.: Studien-Verl. 2003, S. 259-275.

Škreb, Zdenko: Grillparzer. Eine Einführung in das dramatische Werk. Kronberg/Ts.: Scriptor 1976.

Zenko, Franjo: Philosophie an der neugegründeten Universität Zagreb (1874) zur Jahrhundertwende und ihr Verhältnis zur damaligen Schulphilosophie in Wien. In: Ambivalenz des Fin de siècle: Wien - Zagreb. Hgg. Damir Barbarić, Michael Benedikt. Wien, Köln, Weimar: Böhlau 1998, S. 48-66. 